\title{
FLUID ANALYSIS OF MAGNETIZED PLASMA SHEATH IN A CYLINDRICAL GEOMETRY
}

\author{
P. K. Thakur*, R. R. Pokhrel, R. Khanal \\ Central Department of Physics, Tribhuvan University, Kirtipur, Kathmandu, Nepal \\ *Corresponding authore-mail: pk_thakur12@yahoo.com \\ (Received: September 5, 2018; Revised: November 15, 2018; Accepted: November 18, 2018)
}

\begin{abstract}
Plasma sheath formed in front of a material wall plays an important role in overall plasma properties. Magnetized plasma sheath for both collisional and collisionless cases in a cylindrical co-ordinate system was studied using a fluid model. The fluid equations were compiled for the considered geometry and were solved numerically, using the fourth-order RungeKutta method for prescribed boundary and initial conditions. The ion velocity along the axis of the cylinder and the ion density profiles were studied for collisionless and collisional cases and at different obliqueness of the magnetic field. The ion velocities acquired its maximum value at the wall with monotonic increment in collisionless cases, whereas the ion density profile was not monotonic in collisionless case as well as when the obliqueness of the magnetic field starts increasing. In such cases, the ion density increases close to the entrance and then decreases monotonically towards the wall. The study provides insight to plasma properties in cylindrical plasmas which are common in discharge tubes, light sources and plasma jets.
\end{abstract}

Keywords: Plasma, Sheath, Mach number, Bohm-Chodura condition

\section{INTRODUCTION}

The Nobel prize-winning American Chemist Irving Langmuir first used term plasma to describe an ionized gas in analogy to the way blood plasma carries red and white corpuscles by the way an electrified fluid carries electrons and ions (Langmuir 1928). He developed the theory of plasma sheath, the boundary layer which forms between ionized plasma and solid surfaces. He also discovered that certain regions of a plasma discharge tube exhibit periodic variations of the electron density, which is termed as the Langmuir waves. Magnetized plasma sheaths show distinctly different behavior compared to unmagnetized cases and is one of the important research problems in plasma experiments. The pioneering work by Chodura (1982) draw attention of many in this area and the study of magnetized plasma sheath is still one of the important research problems (Riemann 1994, Ahedo 1997, Tskhakaya \& Kuhn 2005, Masoudi et al. 2010, Havlickova \& Hrach 2010, Chalise \& Khanal 2012, 2015, Robertson 2013). In this work, the effect of magnetic field in a cylindrical plasma sheath for both collisional and collisionless cases was studied using the fluid model. The obtained results are in good agreement with previously reported works and are expected to provide better insight in understanding plasma sheath properties especially in cylindrical geometries.

\section{MODEL AND BASIC EQUATION}

A steady state plasma sheath formed inside a cylinder is considered as shown in Fig. 1, where $\mathrm{z}=0$ represents the sheath entrance and plasma is quasi-neutral and $\mathrm{z}=\mathrm{L}$ is the material wall. It is assumed that the plasma consists of hydrogen ions and electrons and they enter the sheath only along the $\mathrm{z}$-direction. The externally applied magnetic field (B) has components along $\mathrm{z}$ and $\rho$ direction as $\mathrm{B} \cos \theta$ and $B \sin \theta$, respectively, and the velocity components are $\mathrm{v}_{\rho}, \mathrm{v}_{\theta}$ and $\mathrm{v}_{\mathrm{z}}$. We assume the plasma to be isothermal, that is, $\mathrm{T}_{\mathrm{e}} \approx \mathrm{T}_{\mathrm{i}}$ where, $\mathrm{T}_{\mathrm{e}}$ and $\mathrm{T}_{\mathrm{i}}$ are electron and ion temperatures, respectively.

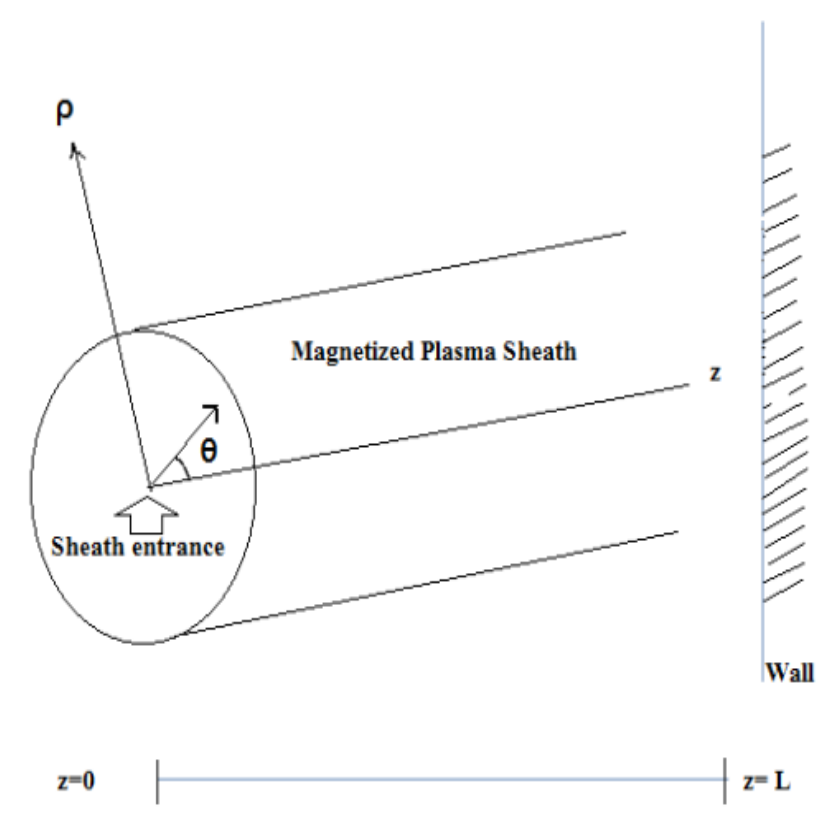

Fig. 1. Magnetized plasma sheath formed in a cylindrical geometry

The following fluid equations are compiled and solved numerically for prescribed initial condition by using the given boundary conditions. The equation of continuity 


$$
\frac{\partial \mathrm{n}_{\mathrm{s}}}{\partial \mathrm{t}}+\nabla \cdot\left(\mathrm{n}_{\mathrm{s}} \overrightarrow{\mathrm{v}}_{\mathrm{s}}\right)=0
$$

Where, $n_{\mathrm{s}}$ and $\overrightarrow{\mathrm{v}}_{\mathrm{s}}$ represent the particle number density and the velocity of the species-s, respectively. We consider momentum equation for the steady state as

$\mathrm{v}_{\mathrm{z}} \frac{\partial \overrightarrow{\mathrm{v}}}{\partial \mathrm{z}}=\frac{\mathrm{e}}{\mathrm{m}_{\mathrm{i}}} \frac{\mathrm{d} \Phi}{\mathrm{dz}} \overrightarrow{\mathrm{z}}+\frac{\mathrm{e}}{\mathrm{m}_{\mathrm{i}}}(\overrightarrow{\mathrm{E}}+\overrightarrow{\mathrm{v}} \times \overrightarrow{\mathrm{B}})-\gamma_{\mathrm{i}} \overrightarrow{\mathrm{v}}$

Where, $\Phi$ and $\gamma_{\mathrm{i}}$ denote the local electrostatic potential and effective ion collision frequency, respectively. We consider

$\gamma_{\mathrm{i}}=\mathrm{n}_{\mathrm{n}} \sigma_{\mathrm{s}} \mathrm{v}_{\mathrm{z}}=\frac{\alpha}{\lambda_{\mathrm{D}}} \mathrm{v}_{\mathrm{z}}$

where, $\alpha$ is a dimensionless parameter which characterizes magnitude of the collision force, $\mathrm{n}_{\mathrm{n}}$ is the neutral gas density, $\sigma_{\mathrm{s}}$ is the cross-section measured at ion acoustic velocity and $\lambda_{\mathrm{D}}$ is the electron Debye length. For the geometry considered (Fig. 1), we obtain

$\rho$-component: $\mathrm{v}_{\mathrm{z}} \frac{\mathrm{dv} \mathrm{v}_{\rho}}{\mathrm{dz}}=\frac{\mathrm{e}}{\mathrm{m}_{\mathrm{i}}} \mathrm{v}_{\theta} \mathrm{B} \cos \theta-\frac{\alpha \mathrm{v}_{\mathrm{z}}}{\lambda_{\mathrm{D}}} \mathrm{v}_{\rho}$

$\theta$-component:

$\mathrm{v}_{\mathrm{z}} \frac{\mathrm{dv}_{\theta}}{\mathrm{dz}}=\frac{\mathrm{e}}{\mathrm{m}_{\mathrm{i}}} \mathrm{B}\left[\mathrm{v}_{\mathrm{z}} \sin \theta-\mathrm{v}_{\rho} \cos \theta\right]-\frac{\alpha \mathrm{v}_{\mathrm{z}}}{\lambda_{\mathrm{D}}} \mathrm{v}_{\theta}$

z-component: $\mathrm{v}_{\mathrm{z}} \frac{\mathrm{dv_{ \textrm {z } }}}{\mathrm{dz}}=-\frac{\mathrm{e}}{\mathrm{m}_{\mathrm{i}}} \frac{\mathrm{d} \Phi}{\mathrm{dz}}-\frac{\mathrm{e}}{\mathrm{m}_{\mathrm{i}}} \mathrm{v}_{\theta} \mathrm{B} \sin \theta-\frac{\alpha \mathrm{v}_{\mathrm{z}}}{\lambda_{\mathrm{D}}} \mathrm{v}_{\mathrm{z}}$

The electrons are assumed to be in thermal equilibrium so they obey Boltzmann distribution:

$\mathrm{n}_{\mathrm{n}}=\mathrm{n}_{0} \exp \frac{\mathrm{e} \Phi}{\mathrm{k}_{\mathrm{B}} \mathrm{T}_{\mathrm{e}}}$

Poisson equation which relates to electrostatic potential to the ion and electron densities:

$\frac{\mathrm{d}^{2} \Phi}{\mathrm{dz}^{2}}=\frac{\mathrm{e}}{\varepsilon_{0}}\left(\mathrm{n}_{\mathrm{e}}-\mathrm{n}_{\mathrm{i}}\right)$

For steady state and using the following normalization

$$
\mathrm{N}_{\mathrm{s}}=\frac{\mathrm{n}_{\mathrm{s}}}{\mathrm{C}_{\mathrm{s}}}, \mathrm{U}_{\mathrm{s}}=\frac{\mathrm{v}_{\mathrm{s}}}{\mathrm{C}_{\mathrm{s}}}, \xi=\frac{\mathrm{z}}{\lambda_{\mathrm{D}}}, \eta=\frac{-\mathrm{e} \Phi}{\mathrm{k}_{\mathrm{B}} \mathrm{T}_{\mathrm{e}}}
$$

and governing fluid equations are

Continuity equation: $\mathrm{N}_{\mathrm{i}} \mathrm{U}_{\mathrm{z}}=\mathrm{M}$

$\rho$-component:

$\frac{\mathrm{dU}}{\mathrm{d} \xi}=\frac{\mathrm{U}_{\theta}}{\mathrm{U}_{\mathrm{Z}}} \beta \cos \theta-\alpha \mathrm{U}_{\rho}$ $\theta$-component: $\frac{\mathrm{d} \mathrm{U}_{\theta}}{\mathrm{d} \xi}=\beta\left[\sin \theta-\frac{\mathrm{U}_{\rho}}{\mathrm{U}_{\mathrm{z}}} \cos \theta\right]-\alpha \mathrm{U}_{\theta}$

Z-component: $\frac{\mathrm{d} \mathrm{U}_{\mathrm{z}}}{\mathrm{d} \xi}=-\frac{1}{\mathrm{U}_{\mathrm{z}}} \frac{\mathrm{d} \eta}{\mathrm{d} \xi}=\frac{\mathrm{U}_{\theta}}{\mathrm{U}_{\mathrm{z}}} \sin \theta-\alpha \mathrm{U}_{\mathrm{z}}$

Equation of motion of electron: $\mathrm{N}_{\mathrm{e}}=\exp (-\eta)$

Poisson equation: $\frac{d^{2} \eta}{d \xi^{2}}=N_{i}-\exp (-\eta)$

Where, $r_{1}=\sqrt{\frac{m_{i} k_{B} T_{e}}{e^{2} B^{2}}}$ is the ion gyro radius, $C_{s}=\sqrt{\frac{k_{B} T_{e}}{m_{i}}}$ is the ion acoustic velocity and $\beta=\frac{\lambda_{\mathrm{D}}}{\mathrm{r}_{1}}=\mathrm{B} \sqrt{\frac{\varepsilon_{0}}{\mathrm{~m}_{\mathrm{i}} \mathrm{n}_{0}}}$ which depends on the magnitude of magnetic field.

\section{RESULTS AND DISCUSSION}

The fluid equations from (10) to (15) are solved numerically using the fourth-order Runge-Kutta method to study the ion velocity profile along the axis of the cylinder and the ion density profiles at different angles. Collision is characterized by $\alpha$ such that $\alpha=0$ refers to the collisionless case. The initial and boundary conditions prescribed are the normalized potential at sheath entrance is 0 , that is, $\eta(\xi=0)=0$; the normalized velocities at sheath entrance are $U_{\rho o}=0, U_{\theta o}=0$ and $U_{z o}=1$; the electric field at sheath entrance $\eta(\xi=0)=0.01$ and the ion Mach number $\mathrm{M}=1$ (to satisfy the Bohm-Chodura condition). Details of the numerical methods and analysis of additional parameters were described elsewhere (Pokhrel 2015).

The z-component of ion velocity as a function of sheath distance for collisionless and collisional cases at three different values of $\theta$ are plotted in Figs 2 and 3, respectively. The ion velocities acquire its maximum value at the wall in all cases. For $\theta=0$ and in collisionless case, the increment is monotonic, however with increasing $\theta$, the ions gyration close to sheath entrance causes the ion velocity to decrease. As the ion move away from the entrance, the electric field starts of dominating and hence the gyration is negligible. But in presence of collision, the effect of magnetic field is dominated by the collisional force and hence no more fluctuation is observed. At $\theta=0, \mathrm{z}$-component of normalized velocity is more for collisionless case than collisional case. According to Poisson equation, the increase of the ion density distribution would lead to increase of the potential. At $\theta=\pi / 2$, z-component of the normalized velocity in collisionless and collisional case increases in nearly same ratio. The potential are also in same ratio. At $\theta=5 \pi / 6$, z-component of velocity acquired higher value in collisonless than in collisional case. 


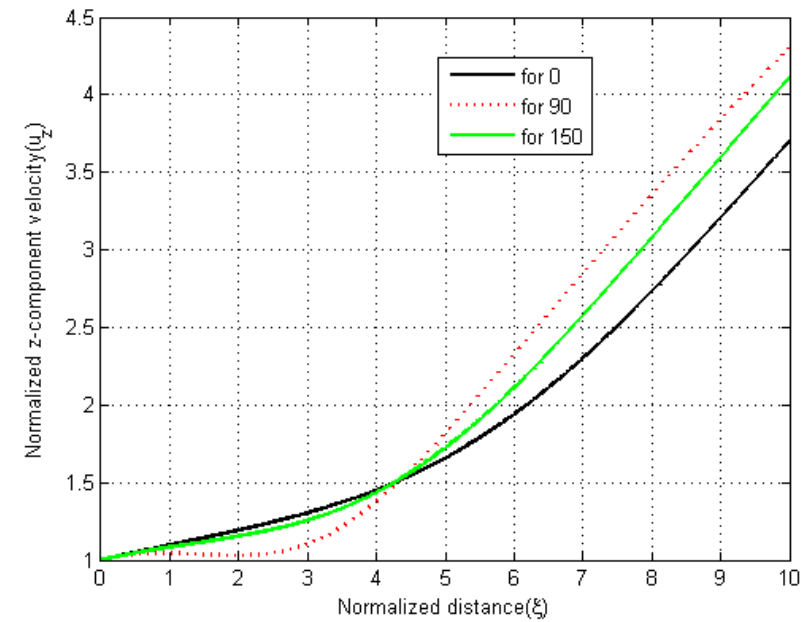

Fig. 2. z-components of ion velocity profile for collisionless case and three different values of $\theta$

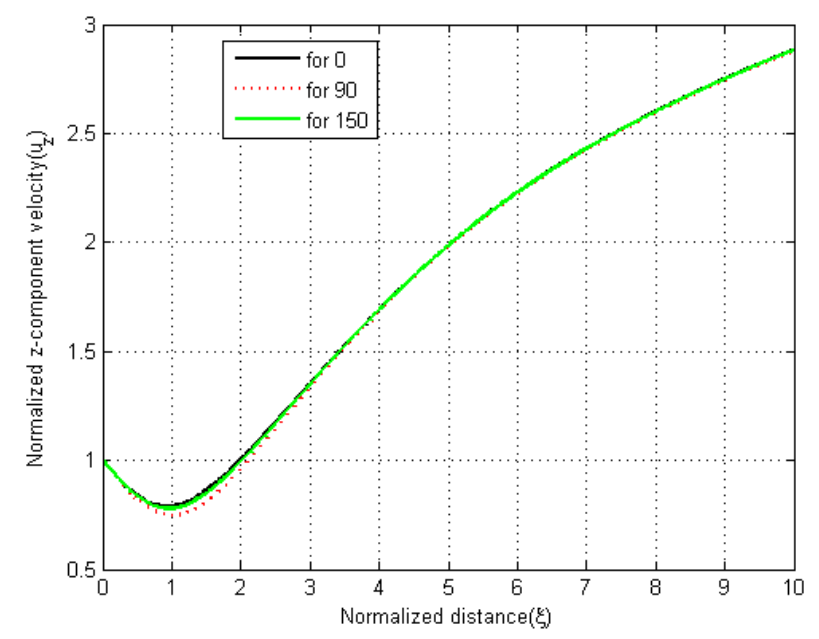

Fig. 3. z-components of ion velocity profile for collisional case and three different values of $\theta$

The ion density profile at angles $\theta=0$ and at $\theta=\pi / 2$ as a function of sheath distance for collisionless and collisional cases are plotted in Figs 4 and 5, respectively. In absence of collision and when the magnetic field is absent (i. e. $\theta=$ 0 and $\mathrm{B}=0$ ) the ion density profile decrease monotonically. The ion density profile is no more monotonic in collisionless case or when the obliqueness of the magnetic field starts increasing.

In such cases, the ion density increases close to the entrance and then decreases monotonically towards the wall. According to the equation of continuity, for increase in the ion density there will be decrease in the ion velocity. The electric field is dominant close to the wall and hence there is less effect of collision and magnetic field. For motions perpendicular to B, the fluid theory is a good approximation. The electrons in a plasma drift with a velocity proportional to $\mathrm{E}$. In the sense, collisionless plasma behaves like a collisional fluid. So that amplitude of ion density is maximum in the collisionless case.

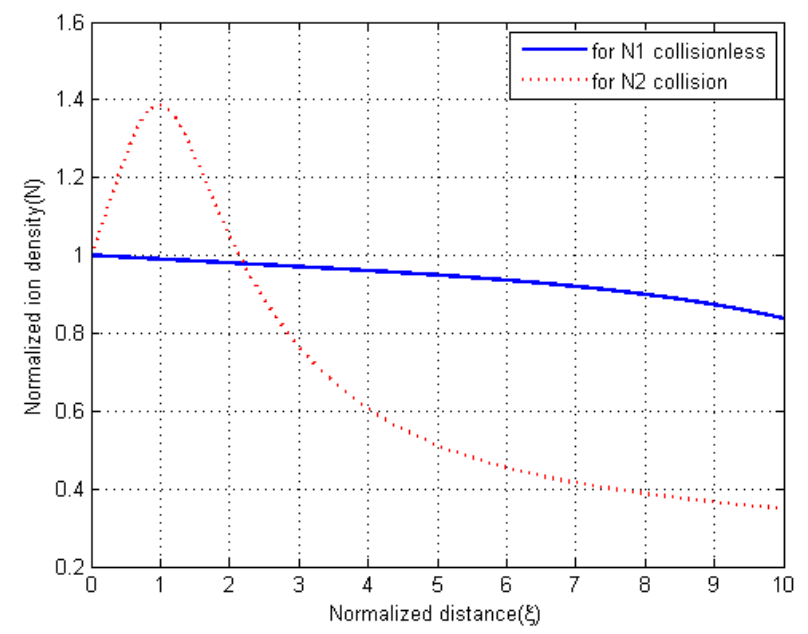

Fig. 4. Ion density profile at an angle $\theta=0$

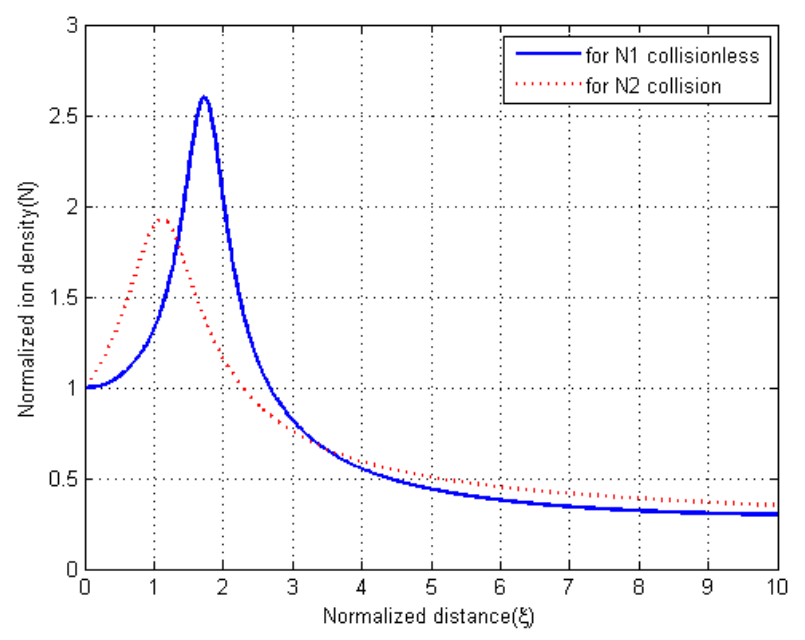

Fig. 5. Ion density profile at an angle $\theta=\pi / 2$

\section{CONCLUSIONS}

The magnetized plasma sheath in a cylindrical co-ordinate system with oblique magnetic field was studied numerically by using the fluid hydrodynamic model. The ion velocities acquire maximum value at the wall in all cases. In the collisional case, the presence of collisional force dominates the effect of magnetic field on the ion velocity. Magnetic field makes the ion density distribution rise at first and drop. The reason for it is that the Lorentez force slow down the ion flow velocity in the z-direction at first. The electric field is dominant close to the wall and hence there is less effect of collision and magnetic field. As the obliqueness of the magnetic field increases, the amplitude of the ion density also increases in both cases. The collision effect cause decrease in ion velocity but to conserve the flux the ion density must increase, it causes increase in wall potentials. 


\section{REFERENCES}

Langmuir, I. 1928. Oscillations in ionized gases. Proceedings of the National Academy of Sciences 14(8): 627-637.

Chodura, R. 1982. Plasma wall transition in an oblique magnetic field. The Physics of Fluids 25: 1628-1633.

Riemann, K. U. 1994. Theory of the plasma-sheath transition in an oblique magnetic field. Contributions to Plasma Physics 34: 127-132.

Ahedo, E. 1997. Structure of the plasma-wall interaction in an oblique magnetic field. Physics of Plasmas 4(12): 4419-4430.

Tskhakaya, D. and Kuhn, S. 2005. Kinetic (PIC) simulations of the magnetized plasma-wall transition. Plasma Physics and Controlled Fusion 47: A327-A337.

Masoudi, S. F., Esmaeili, S S. and Jazavandi, S. 2010. Ion dynamics in plasma sheath under the effect of $\mathrm{E} \times \mathrm{B}$ and collisional forces. Vacuum 84: 382-386.
Havlickova, E. and Hrach, R. 2010. Numerical investigations of sheath structure in presence of magnetic field. Journal of Physics: Conference Series 207: 012030 1-7.

Chalise, R. and Khanal, R. 2012. A kinetic trajectory simulation model for magnetized plasma sheath. Plasma Physics and Controlled Fusion 54: 095006 1-5.

Robertson, S. 2013. Sheaths in laboratory and space plasmas. Plasma Physics and Controlled Fusion 55(9): 093001-34.

Chalise, R. and Khanal, R. 2015. Self-consistent one dimension in space and three dimension in velocity kinetic trajectory simulation model of magnetized plasma-wall transition. Physics of Plasmas 22: 1135051-1135055.

Pokhrel, R.R. 2015. Effect of magnetic field in cylindrical plasma sheath. M. Sc. Dissertation, Tribhuvan University, Kathmandu, Nepal. 